\title{
Coronal Heating by MHD Waves: Results from the SECIS Instrument during the 1999 Eclipse
}

\author{
K. J. H. Phillips ${ }^{1}$, P. T. Gallagher ${ }^{2}$, D. R. Williams ${ }^{2}$, F. P. Keenan ${ }^{2}$, P. \\ Rudawy $^{3}$, B. Rompolt ${ }^{3}$, and A. Berlicki ${ }^{3}$, \\ ${ }^{1}$ Space Science $\&$ Technology Dept., CLRC Rutherford Appleton \\ Laboratory, Chilton, Didcot, Oxon. OX11 OQX, U.K. \\ ${ }^{2}$ Dept. of Physics, Queen's University, Belfast BT7 1NN, N. Ireland, \\ U.K. \\ ${ }^{3}$ Astronomical Institute, University of Wroctaw, ul. M. Kopernika 11, \\ Wroctaw, Poland
}

\begin{abstract}
.
We report on results from the Solar Eclipse Coronal Imaging System (SECIS), an instrument using fast CCD cameras designed to search for short-period modulations in visible-light emission from the corona during an eclipse or with a coronagraph. The instrument was successfully used during the total eclipse of 1999 August 11 from a site in Bulgaria. This paper summarizes both the instrument and preliminary results.
\end{abstract}

\section{Introduction}

The problem of the heating of the solar corona has been highlighted in recent years as a result of $\mathrm{SOHO}$ and other spacecraft. It appears that much of the heating could be attributed to small flare-like events. However, wave heating could still be important, so it is important to search for signatures. Theoretical indications are that high frequency $(>0.5 \mathrm{~Hz})$ MHD waves may be involved in the heating (Porter et al. 1994), higher than what can be observed from spacecraft. Instrumentation must therefore be ground-based at present, and so operate either during total solar eclipses or with coronagraphs (Pasachoff \& Ladd 1987; Koutchmy et al. 1983). Here we report on successful observations made with the Solar Eclipse Coronal Imaging System (SECIS) during the eclipse of 1999 August 11.

\section{The SECIS Instrument and its Performance}

Full instrumental details about the SECIS instrument are given by Phillips et al. (2000) so only a short description is given here. In the set-up for the 1999 eclipse, two fast-frame digital CCD cameras $(512 \times 512$ pixel format) were used to image both the white-light and green-line solar coronal emission. A computer system captures the digitised signal from the cameras and reconstitutes and stores the images. The exposure time and frame frequency are adjustable. A high-precision 
heliostat directing light from the eclipsed Sun into the horizontally mounted instrument was designed and constructed at the Astronomical Institute, Wrocław. It has a $250 \mathrm{~mm}$ mirror, flat to $1 / 10$ wave. A $200 \mathrm{~mm}$ Meade Schmidt-Cassegrain $f / 10$ telescope received light from the heliostat. Other optical components split the light into two, one passing through a green-line $(530.3 \mathrm{~nm})$ interference filter, the other with no filter.

Our eclipse site was Shabla, Bulgaria, on the Black Sea coast. We set the camera exposure time to be $20 \mathrm{~ms}$ and frame frequency $44 \mathrm{~s}^{-1}$. During the eclipse, the sky was almost completely cloudless and during totality there was no wind. We recorded 12728 images, 6364 from each of the two cameras. The field of view $\left(0.5^{\circ} \times 0.5^{\circ}\right)$ was selected from $S O H O$ images. The equipment operated flawlessly apart from a slight image drift. After the eclipse, darkcurrent and flat-fielding images were taken to enable corrections to be made to the observations.

\section{Results and Preliminary Analysis}

Fig. 1 shows one of the images obtained from the white-light channel. As well as coronal features several bright prominences are evident enabling the images to be correctly orientated. The data were written to FITS files for easier analysis. Corrections were made for the image drift by one of us (PR) using the small prominence on the Sun's south-west limb and the moving disk of the Moon. The spatial scale established, 1 pixel $=4.04$ arcsecs, agrees almost perfectly with pre-eclipse measurements. The spatial resolution, determined by seeing, is about equal to 1 pixel. A routine was written to allow for the image motion, as well as dark-current subtraction and flat-fielding.

Analysis of the resulting corrected green-line images has proceeded along two separate lines of investigation: PR has used a standard Fourier technique, using the commercial Statistica software package and own software writen in IDL, and DRW has used a wavelet analysis similar to those now extensively used in $\mathrm{SOHO}$ data with IDL procedures. In PR's analysis, the power spectrum and spectral density of the signal were calculated using a Fourier transformation algorithms, generally on selected single pixels in the images. About 400 pixels have been examined so far. To minimize the effect of the image drift mentioned above, the images were numerically shifted to the common reference system with the accuracy better than 0.1 of pixel. To minimize the effect of image rotation caused by use of the single-mirror heliostat, the data were grouped into time periods of 1000 time points (about 23s). The method is thus sensitive to frequencies $>1 \mathrm{~Hz}$. Most points analyzed show little evidence of oscillatory behaviour, which would suggest MHD wave heating. However, a small number of pixels have been found for which the signal has oscillatory behaviour with significant power for some frequencies. These points are generally located in identifiable coronal structures such as the tops of coronal loops. Power spectra and spectral density plots of these points show large peaks at frequencies between 8 and $11 \mathrm{~Hz}$. Fisher's test (see e.g. Shimshoni 1971) shows significance levels of a little larger than $90 \%$ for these peaks. The data were examined using the IDL wavelet analysis procedure. The Fourier analysis for points showing strong peaks is confirmed, the wavelet analysis revealing the periodicity as a band of 


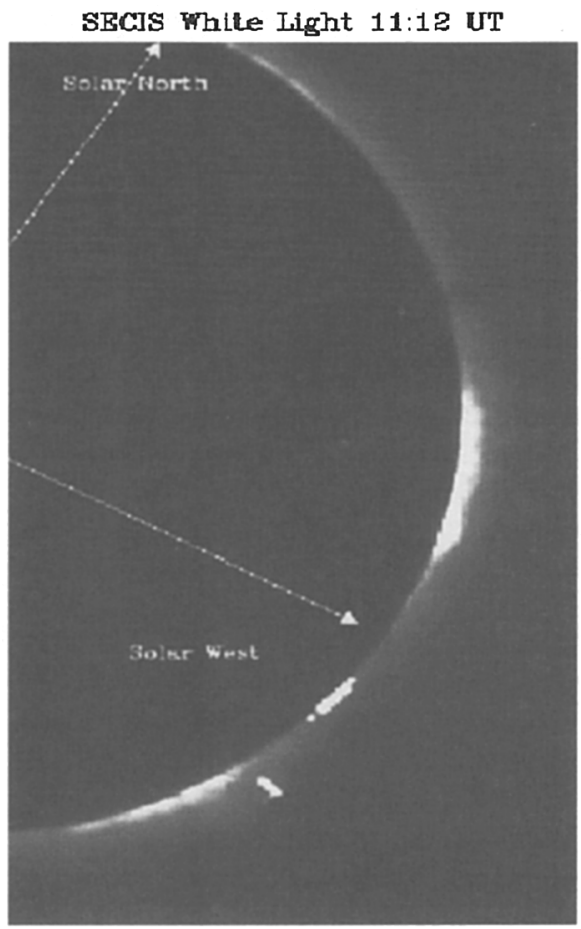

Figure 1. One of the 6364 white-light images taken by SECIS at approximately mid-totality during the eclipse of 1999 August 11 . The field of view covers the solar limb from just east of the north point to south of the west point. The image includes both coronal features and prominences. 
enhanced power across much the plot of frequency vs. time produced by the wavelet procedure.

\section{Preliminary Conclusions}

With such an enormous amount of data to correct and analyze, it is difficult to draw any definite conclusions at this early stage, but it is looking as if there is no strong evidence for oscillatory behaviour in the green-line emission in the SECIS images except for isolated points. Analysis is continuing, so more definite conclusions should be arrived at soon.

\section{Acknowledgements}

We thank Prof. V. N. Dermendjiev, Academician Prof. D. Mishev, and Dr Maria Madjarska for arranging the eclipse site and despatch of our equipment. PR, BR and $\mathrm{AB}$ were supported by the grant no. 2PO3D00515 of the Polish Committee of Scientific Research. The UK groups are supported by the Leverhulme Trust.

\section{References}

Pasachoff, J. M., \& Ladd, E. F. 1987. Solar Phys. 109, 365

Phillips, K.J.H. et al. 2000. Solar Phys. 193, 259

Porter, L.J., Klimchuk, J.A., \& Sturrock, P.A. 1994. ApJ, 435, 482

Shimshoni, M. 1971. Geophys. J. Roy. Astr. Soc., 23, 373 